\title{
Monte Carlo calculations of nucleon-induced fission in the GeV energy range
}

\author{
Sergio Lo Meo ${ }^{1,4, a}$, Davide Mancusi ${ }^{2, b}$, Cristian Massimi ${ }^{3,4, \mathrm{c}}$, Diego Tarrio ${ }^{5, \mathrm{~d}}$, Gianni Vannini ${ }^{3,4, \mathrm{e}}$, and Alberto Ventura ${ }^{4, \mathrm{f}}$ \\ 1 ENEA, Centro Ricerche Ezio Clementel, Bologna, Italy \\ 2 Den - Service d' étude des réacteurs et de mathématiques appliquées (SERMA), CEA, Université Paris-Saclay, 91191 Gif-sur-Yvette, \\ France \\ 3 Dipartimento di Fisica e Astronomia, Università di Bologna, Italy \\ ${ }^{4}$ Istituto Nazionale di Fisica Nucleare, Sezione di Bologna, Italy \\ 5 Uppsala University, Dept. of Physics and Astronomy, Uppsala, Sweden
}

\begin{abstract}
In the present work the Monte Carlo calculations of nucleon-induced fission on actinides and preactinides from $100 \mathrm{MeV}$ to $1 \mathrm{GeV}$, described in a recent paper of ours [1], are extended up to an incident energy of $8 \mathrm{GeV}$. The calculated $(p, f)$ cross sections are compared with the few experimental data available in the literature. The same model parameters are used to predict $(n, f)$ cross sections in the same energy range. Possible shortcomings of decay models at high incident energies are discussed.
\end{abstract}

\section{Introduction}

Fission induced by nucleons at intermediate energies is frequently described as a three-stage process: a fast cascade phase, a pre-equilibrium phase and a final evaporation-fission of equilibrated heavy remnants produced by the fast processes. In the Liège Intranuclear Cascade Model, INCL++, used in in our previous paper [1] in the energy range from $100 \mathrm{MeV}$ to $1 \mathrm{GeV}$ and in the present work from $100 \mathrm{MeV}$ to $8 \mathrm{GeV}$, a self-consistent determination of the stopping time of the fast cascade [2] makes it possible to skip the pre-equilibrium stage, so as to reduce intermediate energy fission to a twostage process. In this work use is made of version 5.2 [3] of INCL++, including multiple pion production as described in Ref. [4], and thus allowing calculations up to $10-12 \mathrm{GeV}$, while version 5.1.14 adopted in our previous work [1] described only single pion production from the decay of delta resonances; the versions of the evaporationfission models yielding fission cross sections, GEMINI++ [5] and ABLA07 [6], are the same as in Ref. [1], so that the changes of input parameters and of numerical results below $1 \mathrm{GeV}$ are entirely connected with the new version of the fast cascade model. The studied energy range is limited to $8 \mathrm{GeV}$ because of possible shortcomings of decay models at higher energies, to be discussed later.

a e-mail: sergio.lomeo@enea.it

b e-mail: davide.mancusi@cea.fr

c e-mail: cristian.massimi@bo.infn.it

d e-mail: diego.tarrio@physics.uu.se

e e-mail: gianni.vannini@bo.infn.it

f e-mail: alberto.ventura@bo.infn.it

\section{Cross section calculations}

Following the philosophy of the authors of INCL++, as we already did in Ref. [1], no parameters have been modified in the intranuclear cascade model, already optimized by reproducing a large amount of data connected with the cascade stage, while two basic fission parameters have been taken as adjustable in the decay models, i.e. the asymptotic level density parameter of the fission channel, $a_{f}$, multiplied by a factor $k$ in ABLA07, or the ratio of $a_{f}$ to the level density parameter of the neutron channel, $a_{n}$, in GEMINI++, where the default value is $a_{f} / a_{n}=$ 1.036, and the height of the fission barriers, $B_{f}$ of the remnants, that can be reduced, or increased by the same amount, $\Delta B_{f}$.

This is, admittedly, a rather crude approximation, aiming at reproducing experimental fission cross sections of single nuclei and it is not extensible to nuclei for which no data exist. Moreover, no attempt is made to reproduce experimental mass and charge distributions of fission fragments, or neutron emission during the fission process, where transient and dissipative effects are known to play an important role: in recent years, researchers from the University of Santiago de Compostela and their collaborators attempted a similar-in-spirit comprehensive description of proton-induced fission cross sections [7-10]. In their approach, mostly focused on INCL/ ABLA07, they find that a coherent description of several observables in proton-induced fission reactions up to $1 \mathrm{GeV}$ is possible with a choice of physically grounded ABLA07 parameters. In particular, level densities at ground-state and saddle-point deformations are fixed by means of the measured widths of the charge distributions of final fission fragments [9] and the reduced dissipation parameter by means of pre-scission neutron multiplicities and average neutron excess of final fission fragments [11]. 
Table 1. Adjusted model parameters.

\begin{tabular}{lllll}
\hline Isotope & ${ }^{238} \mathrm{U}$ & ${ }^{235} \mathrm{U}$ & ${ }^{197} \mathrm{Au}$ & ${ }^{181} \mathrm{Ta}$ \\
\hline$a_{f} / a_{n}(\mathrm{GEMINI})$ & 1.039 & 1.045 & 1.043 & 1.041 \\
\hline$\Delta B_{f}(\mathrm{MeV})(\mathrm{GEMINI})$ & -0.3 & -0.2 & 0.0 & 0.0 \\
\hline$k(\mathrm{ABLA07})$ & 1.020 & 1.023 & 1.010 & 1.012 \\
\hline$\Delta B_{f}(\mathrm{MeV})(\mathrm{ABLA07})$ & -0.3 & -0.2 & -0.1 & 0.0 \\
\hline
\end{tabular}
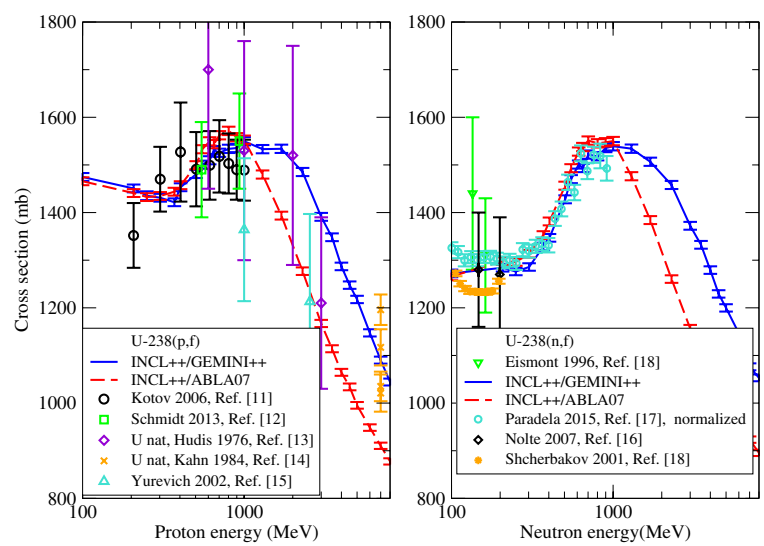

Figure 1. Left panel: ${ }^{238} U(p, f)$. Solid line: GEMINI calculation; dashed line: ABLA07 calculation. Right panel: ${ }^{238} U(n, f)$. Solid line: GEMINI; dashed line: ABLA07.

Our results of fission cross section calculations are shown for an actinide, ${ }^{238} U$, and two pre-actinides, ${ }^{197} \mathrm{Au}$ and ${ }^{181} \mathrm{Ta}$. Table I lists the model parameters adjusted on $(p, f)$ data, i.e. $a_{f} / a_{n}$ and $\Delta B_{f}$ (in MeV) for GEMINI++, $k$ and $\Delta B_{f}$ for ABLA07, for the above mentioned nuclides and for ${ }^{235} U$, not discussed in the present work, but used in the normalization of an experimental ${ }^{238} U(n, f)$ cross section.

\section{1. ${ }^{238} \mathrm{U}$}

Recent reliable $(p, f)$ data of ${ }^{238} U$ are available below $1 \mathrm{GeV}[12,13]$, while, in the range from 1 to $8 \mathrm{GeV}$, older data of ${ }^{n a t} U$ exist $[14,15]$, which appear to be compatible with the above mentioned data below $1 \mathrm{GeV}$, while other recent data of ${ }^{238} U$ from 1 to $3 \mathrm{GeV}$ [16] are systematically lower. Among $(n, f)$ experiments, a prominent role is played by Ref. [17], since it provides a measurement of the absolute cross section, while the majority of authors measure the ratio to the ${ }^{235} U(n, f)$ cross section, whose absolute values, however, are not experimentally known beyond $200 \mathrm{MeV}$. In the case of Ref. [18], whose relative fission data extend up to $1 \mathrm{GeV}$, we have normalized them to a theoretical ${ }^{235} U(n, f)$ cross section (I. Duran et al., these proceedings), calculated with the same codes used in the present work: the ${ }^{238} U(n, f) /{ }^{235} U(n, f)$ ratios adopted in the present work are the weighted averages of the four measurements described in Ref. [18] (last column in Table IV of the quoted paper). No attempt has been made to renormalize the data of older experiments, taken from the EXFOR library [19].

Figure 1 shows on the left panel $(p, f)$ cross sections calculated with both chains, INCL++/GEMINI++ and INCL++/ABLA07, in comparison with experimental data: below $1 \mathrm{GeV}$ the two chains provide similar fits, above $1 \mathrm{GeV}$, ABLA07 results are systematically lower than GEMINI++, but the large uncertainties of the few experimental data do not allow us to discriminate between
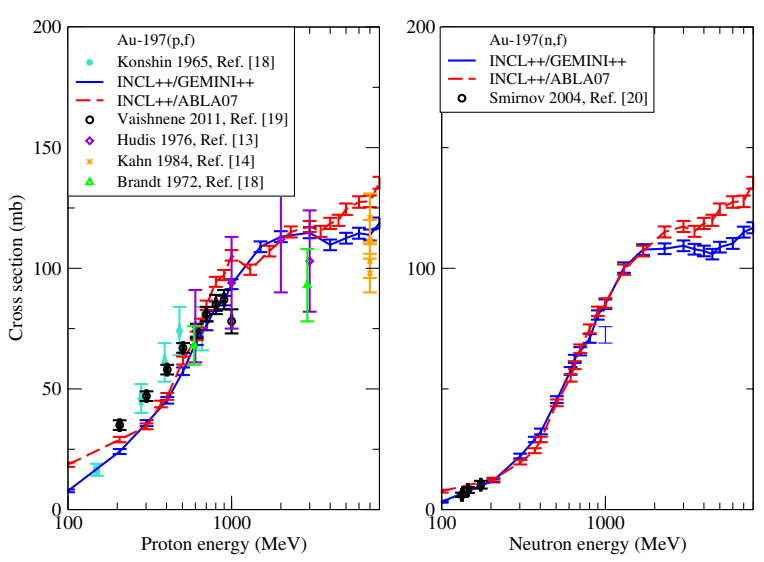

Figure 2. Left panel: ${ }^{197} A u(p, f)$. Solid line: GEMINI calculation; dashed line: ABLA07 calculation. Right panel: ${ }^{197} A u(n, f)$. Solid line: GEMINI; dashed line: ABLA07.

the two models. On the right panel both the calculated $(n, f)$ cross sections compare well with the data of Ref. [17] below $200 \mathrm{MeV}$ and the normalized data of Ref. [18] up to $1 \mathrm{GeV}$. Like in the $(p, f)$ case, the theoretical $(n, f)$ curves show increasing discrepancies beyond $1 \mathrm{GeV}$, where no data exist: there, the ABLA07 curve decreases faster than the GEMINI++ one with increasing incident energy. It is to be stressed that beyond $1 \mathrm{GeV}$ the $(n, f)$ and $(p, f)$ cross sections calculated in the frame of a given decay model, either GEMINI+ or ABLA07, become closer and closer with increasing nucleon energy.

\section{2. ${ }^{197} \mathrm{Au}$}

Several consistent sets of $(p, f)$ data exist for ${ }^{197} A u$ below $1 \mathrm{GeV}$, and the most recent ones are those of Ref. [20], while above $1 \mathrm{GeV}$ the basic experiments are those of Refs. $[14,15]$, like in the uranium case. As shown on the left panel of Fig. 2, our calculations reproduce the high energy data at the cost of underestimating the data below $500 \mathrm{MeV}$. The energy trend is quite different from the uranium case, since the calculated cross sections increase with increasing proton energy. In the range from $1 \mathrm{GeV}$ to $3 \mathrm{GeV}$, ABLA07 and GEMINI++ appear to be in fair agreement, while at higher energies the ABLA07 curve increases faster. The same model parameters are used to compute the $(n, f)$ cross section, shown on the right panel in comparison with the data of Ref. [21], normalized by the authors to the standard ${ }^{238} U(n, f)$ cross section, which are well reproduced.

\section{3. ${ }^{181} \mathrm{Ta}$}

Here again, consistent sets of $(p, f)$ data below $1 \mathrm{GeV}$ are available from recent experiments [8,22,23], while no data exist at higher energies: in particular, Ref. [8] describes measurements done by the inverse kinematic technique and shows fits of the measured $(p, f)$ cross sections obtained by means of different intranuclear cascade models, including an older version of INCL++ coupled to ABLA07 and GEMINI++, with the latter model working much less satisfactorily than the former. On the contrary, the $(p, f)$ fits we obtain with the two models are in mutual agreement and reproduce the data up to $1 \mathrm{GeV}$ fairly well. At higher energies, the calculated cross 

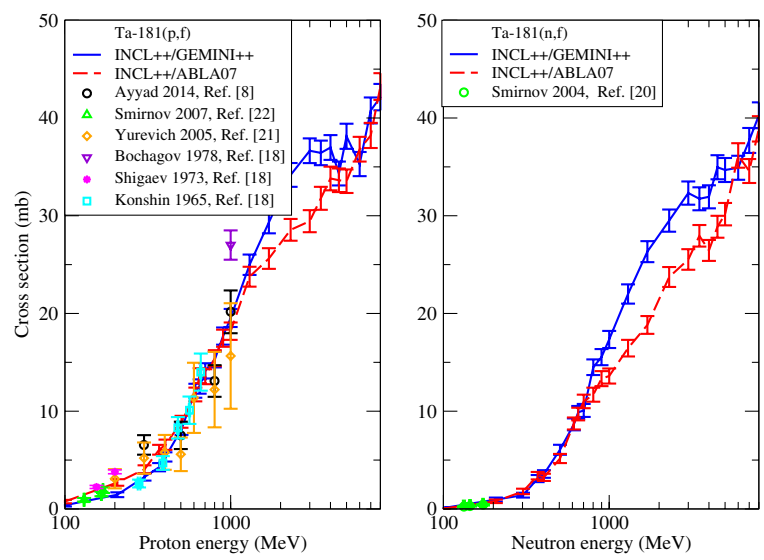

Figure 3. Left panel: ${ }^{181} \operatorname{Ta}(p, f)$. Solid line: GEMINI calculation; dashed line: ABLA07 calculation. Right panel: ${ }^{197} A u(n, f)$. Solid line: GEMINI; dashed line: ABLA07.
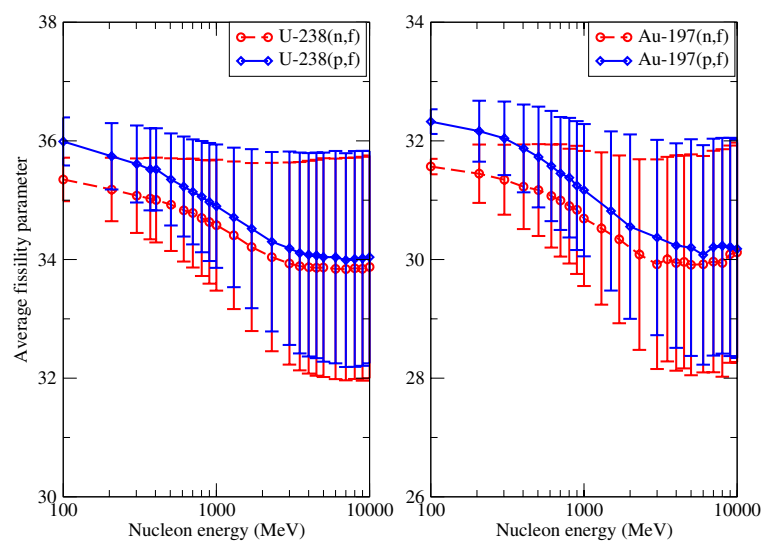

Figure 4. Average remnant fissility parameter vs. incident energy $(\mathrm{MeV})$ : solid curves: $(p, f)$ reactions, GEMINI++ calculations; dashed curves: $(n, f)$ reactions, GEMINI++ calculations. Left panel: ${ }^{238} U$. Right panel: ${ }^{197} A u$.

sections increase quickly and, differently from the ${ }^{197} \mathrm{Au}$ case, the GEMINI++ results are larger than those of ABLA07. The only $(n, f)$ data are again from Ref. [21] and are well reproduced by both models.

The fact that the $(n, f)$ cross section of a given isotope is smaller than the $(p, f)$ cross section below $1 \mathrm{GeV}$ but tends to the same value with increasing incident energy is easily interpreted in terms of the average fissility parameter, $\left\langle Z^{2} / A\right\rangle$, of the equilibrated remnants undergoing fission after the end of the fast cascades, shown in Fig. 4 as a function of incident nucleon energy for ${ }^{238} U$ on the left panel and ${ }^{197} \mathrm{~A} u$ on the right panel. The averages are calculated on fission events only, with the error bars representing the standard deviation of the distribution at each incident energy. In both cases the neutron-induced fissility tends from below to the proton-induced fissility at large incident energy.

Moreover, our calculations show that actinide and preactinide fission cross sections have a similar energy trend up to $1 \mathrm{GeV}$, but in the range from 1 to $8 \mathrm{GeV}$ actinide cross sections decrease and pre-actinide cross sections increase. A qualitatively different behaviour of actinides and pre-actinides was already pointed out in the $(p, f)$ systematics of Ref. [24], where analytic best fits to all the reliable cross section data up to $30 \mathrm{GeV}$ available at

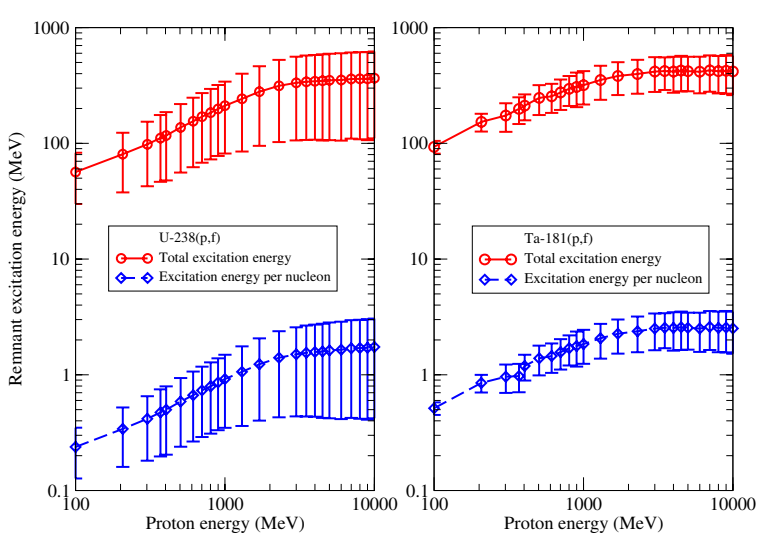

Figure 5. Average remnant excitation energy $(\mathrm{MeV})$ vs. incident energy (MeV): solid curves: total excitation energy; dashed curves: excitation energy per nucleon. Left panel: ${ }^{238} U(p, f)$ GEMINI++ calculation. Right panel: ${ }^{181} T a(p, f)$ GEMINI++ calculation.

that time indicated a much faster decrease with increasing energy for actinides than for pre-actinides. In the ${ }^{238} U$ case, however, the analytic approximation to the $(p, f)$ cross section worked out in Ref. [24] is not consistent with more recent data $[12,13]$ below $1 \mathrm{GeV}$ and decreases much faster than our calculated cross section above $1 \mathrm{GeV}$, which is compatible with the original data of Refs. $[14,15]$. In the cases of ${ }^{197} \mathrm{Au}$ and ${ }^{181} \mathrm{Ta}$, even the most recent $(p, f)$ data below $1 \mathrm{GeV}$ are nicely reproduced by the systematics of Ref. [24], whose cross sections, however, show very modest changes beyond $1 \mathrm{GeV}$, in contrast with our results, which increase with increasing incident energy.

The behaviour of calculated pre-actinide cross sections in the $\mathrm{GeV}$ energy range deserves discussion: a clue might be given by the average excitation energy of the remnants undergoing fission, or, even better, by their average excitation energy per nucleon as a function of incident energy, shown in Fig. 5 for the INCL++/GEMINI++ calculation of the $(p, f)$ cross section of ${ }^{238} U$ on the left panel and ${ }^{181} \mathrm{Ta}$ on the right panel; the statistics used to estimate average values and root-mean-square deviations are limited to fission events in the incident energy range from $100 \mathrm{MeV}$ to $10 \mathrm{GeV}$. In spite of the large deviations, the average excitation energies per nucleon are systematically larger for ${ }^{181} \mathrm{Ta}$ than ${ }^{238} \mathrm{U}$ : for instance, at an incident proton energy of $10 \mathrm{GeV}$, the values are $2.56 \pm 1.0 \mathrm{MeV} /$ nucleon for the former and $1.74 \pm 1.32 \mathrm{MeV} /$ nucleon for the latter. At excitation energies that exceed $3 \mathrm{MeV} /$ nucleon the decay models used in the calculations might need significant revisions: in particular, Bethe-like formulae of nuclear level densities yielding an indefinite increase with energy are no more realistic, since, at excitation energies of the order of $4 \mathrm{MeV} /$ nucleon, level densities are expected to turn over and decrease to zero at a large but finite energy [2527]. Moreover, the competition between fission and multifragmentation, present in the ABLA07 model, but not in GEMINI++, deserves a dedicated study. In this respect, an important role is played by the formation of intermediate mass fragments (IMFs) [28]: a recent dynamical study [29] has shown that the formation of few IMFs can be confused with asymmetric fission if the analysis is limited to yield observables, but from 
a dynamical point of view it can be described as a fragmentation process successively frustrated by meanfield resilience: as an extreme situation, the number of fragments in the exit channel may reduce to two and a fission-like event is obtained by reaggregation.

\section{Conclusions}

Both decay models, ABLA07 and GEMINI++, coupled to INCL++, are able to reproduce $(p, f)$ data in the energy range from $100 \mathrm{MeV}$ to $8 \mathrm{GeV}$ : very close cross sections are obtained below $1 \mathrm{GeV}$, while in $1-8 \mathrm{GeV}$ region the two models give significantly different predictions; the few $(p, f)$ data are not accurate enough to discriminate between the two models. $(n, f)$ cross sections calculated with the same parameters turn out to be in good agreement with experimental data below $1 \mathrm{GeV}$.

\section{References}

[1] S. Lo Meo et al., Nucl. Phys. A 933, 43 (2015)

[2] A. Boudard et al., Phys. Rev. C 66, 044615 (2002)

[3] D. Mancusi et al., Phys. Rev. C 90, 054602 (2014)

[4] S. Pedoux and J. Cugnon, Nucl. Phys. A 866, 16 (2011)

[5] R.J. Charity, Proc. of the Joint ICTP-IAEA Workshop on Model Codes for Spallation Reactions, ICTP Trieste, Italy, 4-8 February 2008, IAEA report INDC(NDS)-530, 139 (2008)

[6] A. Kelič, M.V. Ricciardi and K.H. Schmidt, Workshop of Ref. [5], 181 (2008)

[7] J.L. Rodriguez-Sanchez et al., Phys. Rev. C 90, 064606 (2014)

[8] Y. Ayyad et al., Phys. Rev. C 89, 054610 (2014)
[9] J.L. Rodriguez-Sanchez et al., Phys. Rev. C 92, 044612 (2015)

[10] Y. Ayyad et al., Phys. Rev. C 91, 034601 (2015)

[11] J.L. Rodriguez-Sanchez et al., Phys. Rev. C 94, 061601 (2016)

[12] A.A. Kotov et al., Phys. Rev. C 74, 034605 (2006)

[13] K.-H. Schmidt et al., Phys. Rev. C 87, 034601 (2013)

[14] J. Hudis and S. Katcoff, Phys. Rev. C 13, 1961 (1976)

[15] H.A. Kahn and N.A. Kahn, Phys. Rev. C 29, 2199 (1984)

[16] V.I. Yurevich et al., Phys. Atomic Nuclei 65, 1383 (2002)

[17] R. Nolte et al., Nucl. Sci. Eng. 156, 197 (2007)

[18] C. Paradela et al., Phys. Rev. C 91, 024602 (2015)

[19] www.nndc.bnl.gov/exfor/exfor.htm

[20] L.A. Vaishnene et al., Phys. Atomic Nuclei 74, 115 (2011)

[21] A.N. Smirnov et al., Phys. Rev. C 70, 054603 (2004)

[22] V.I. Yurevich et al., Phys. Part. Nuclei Lett. 2, 49 (2005)

[23] A.N. Smirnov et al., Proceedings of the ND2007 Conference, Nice, 2007, EDP Sciences 2, 1095 (2008)

[24] A.V. Prokofiev, Nucl. Instr. Meth. Phys. Res. A 463, $162(2001)$

[25] M.G. Mustafa et al., Phys. Rev. C 45, 1078 (1992)

[26] S.M. Grimes, Z. Phys. A 343, 125 (1992)

[27] A. Pálffy and H.A. Weidenmüller, Nucl. Phys. A 917, 15 (2013)

[28] P. Napolitani et al., J. Phys. G 38, 115006 (2011)

[29] P. Napolitani and M. Colonna, Phys. Rev. C 92, 034607 (2015) 